\title{
Profiling and targeting of cellular bioenergetics: inhibition of pancreatic cancer cell proliferation
}

\author{
G Cheng ${ }^{1}$, J Zielonka ${ }^{1}$, D McAllister ${ }^{1,2}$, S Tsai ${ }^{3}$, M B Dwinell ${ }^{2}$ and B Kalyanaraman ${ }^{*}, 1$ \\ ${ }^{1}$ Department of Biophysics and Free Radical Research Center, Medical College of Wisconsin, Milwaukee, WI 53226, USA; \\ ${ }^{2}$ Department of Microbiology and Molecular Genetics, Medical College of Wisconsin, Milwaukee, WI 53226, USA and \\ ${ }^{3}$ Department of Surgery/Surgical Oncology, Medical College of Wisconsin, Milwaukee, WI 53226, USA
}

Background: Targeting both mitochondrial bioenergetics and glycolysis pathway is an effective way to inhibit proliferation of tumour cells, including those that are resistant to conventional chemotherapeutics.

Methods: In this study, using the Seahorse 96-well Extracellular Flux Analyzer, we mapped the two intrinsic cellular bioenergetic parameters, oxygen consumption rate and proton production rate in six different pancreatic cancer cell lines and determined their differential sensitivity to mitochondrial and glycolytic inhibitors.

Results: There exists a very close relationship among intracellular bioenergetic parameters, depletion of ATP and anti-proliferative effects (inhibition of colony-forming ability) in pancreatic cancer cells derived from different genetic backgrounds treated with the glycolytic inhibitor, 2-deoxyglucose (2-DG). The most glycolytic pancreatic cancer cell line was exquisitely sensitive to 2-DG, whereas the least glycolytic pancreatic cancer cell was resistant to 2-DG. However, when combined with metformin, inhibitor of mitochondrial respiration and activator of AMP-activated protein kinase, 2-DG synergistically enhanced ATP depletion and inhibited cell proliferation even in poorly glycolytic, 2-DG-resistant pancreatic cancer cell line. Furthermore, treatment with conventional chemotherapeutic drugs (e.g., gemcitabine and doxorubicin) or COX-2 inhibitor, celecoxib, sensitised the cells to 2-DG treatment.

Conclusions: Detailed profiling of cellular bioenergetics can provide new insight into the design of therapeutic strategies for inhibiting pancreatic cancer cell metabolism and proliferation.

Recent research efforts are focused on developing compounds that inhibit tumour cell metabolism and cellular bioenergetics or related molecular pathways that selectively starve tumour cells of essential nutrients capable of generating ATP (Pelicano et al, 2006; Dwarakanath and Jain, 2009; Cheng et al, 2012, 2013). However, targeting tumour cell metabolism has several caveats (DeBerardinis et al, 2007; DeBerardinis and Thompson, 2012). Metabolic phenotype and growth pattern in tumour cells, especially within solid tumours, are totally dependent on several microenvironmental factors including hypoxia, nutrient availability, and angiogenesis (Dang, 2012; Metallo et al, 2012). Tumour cells cope with changing energetic requirements and demands for maintenance and growth via metabolic reprogramming through altered signalling and facilitation of unique metabolic strategies (Ferreira et al, 2012; Ward and Thompson, 2012). One of the first biochemical differences observed in tumour cells as compared with normal cells is a shift in energy metabolism from oxidative phosphorylation (OXPHOS) to aerobic glycolysis, also known as the Warburg effect (Gatenby and Gillies, 2004; Vander Heiden et al, 2009). In addition, enhanced glutamine utilisation and/or fatty-acid oxidation also serve as alternate sources of fuel in tumour cells (Scott et al, 2011). New therapeutic approaches that target multiple bioenergetic pathways in combination with conventional, 'standard-of-care' chemotherapeutics in tumour cells have recently emerged (Tennant et al, 2010; Wise and Thompson, 2010). 
Pancreatic cancer remains one of the most deadly forms of cancer (Hartman and Krasinskas, 2012; American Cancer Society, 2013). There are no effective drug treatments currently available. Thus, new approaches and targeted therapies are desperately required (Strimpakos et al, 2008; Hidalgo, 2010). Identifying specific nutrients that fuel and sustain pancreatic cancer cell growth and selectively inhibiting their uptake by targeting their metabolic pathways present new challenges and opportunities (Regel et al, 2012). Despite the recognition of the Warburg effect in cancer cell bioenergetics, the application of glycolytic inhibitors gave mixed results. Although the origins of the failure of antiglycolytic inhibition are not completely understood, the patientto-patient variability of tumour bioenergetic status may be one of the reasons. Therefore, the new approach of personalised medicine, based on individual profiling of cancer cell bioenergetics, may be a viable strategy for improved efficacy of the existing therapeutics.

In this study, we investigated pancreatic cancer cell metabolism using the Seahorse 96-well Extracellular Flux Analyzer that allows non-invasive real-time monitoring of glycolytic and mitochondrial energetic profiles. To modify pancreatic cancer cell bioenergetics and metabolism, we used 2-deoxyglucose (2-DG) - the US FDAapproved anti-glycolytic agent (Dowling et al, 2011; Iliopoulos et al, 2011; Heinemann et al, 2012; Viollet et al, 2012). Results from this study indicate that there exists a very close relationship between intracellular bioenergetic profiles (rates of OXPHOS and glycolysis) and the susceptibility of cells to glycolytic inhibition in human pancreatic cancer cell culture model systems originating from different genetic backgrounds. The combination of 2-DG with metformin, inhibitor of mitochondrial respiration and activator of AMP-activated protein kinase, lead to enhanced ATP depletion and inhibition of cell proliferation in 2-DG-resistant pancreatic cancer cell line. Thus, we propose that multiple targeting of mitochondrial, glycolytic, and glutaminolytic metabolism and bioenergetics is a viable therapeutic approach to inhibit proliferation of poorly glycolytic, as well as the highly glycolytic, pancreatic cancer cells.

\section{MATERIALS AND METHODS}

Chemicals. 2-Deoxyglucose, metformin, gemcitabine, and doxorubicin were purchased from Sigma-Aldrich (St Louis, MO, USA). Celecoxib was obtained from Cayman Chemical (Ann Arbor, MI, USA) and the stock solution in ethanol $(0.1 \mathrm{M})$ was made freshly as per the manufacturer's instructions. Metformin and 2-DG were freshly made by dissolving the powder in the culture medium as needed, whereas gemcitabine and doxorubicin were dissolved in water as $10 \mathrm{~mm}$ stock and stored in $-80^{\circ} \mathrm{C}$.

Cell culture. The pancreatic cancer cell lines AsPC-1, MiaPaCa-2, PANC-1, HPAF-II, Capan-1, and Capan-2 were acquired from the American Type Culture Collection (Manassas, VA, USA), where they are regularly authenticated. Cells were stored in liquid nitrogen and used within 6 months after thawing. Cell lines were grown at $37^{\circ} \mathrm{C}$ in $5 \% \mathrm{CO}_{2}$ and cultured in DMEM (Invitrogen, Grand Island, NY, USA; cat. no. A14430, supplemented with $5.5 \mathrm{~mm}$ glucose and $4 \mathrm{~mm}$ L-glutamine, but without pyruvate), $10 \%$ fetal bovine serum, penicillin $\left(100 \mathrm{U} \mathrm{ml}^{-1}\right)$ and streptomycin $\left(100 \mu \mathrm{g} \mathrm{ml}^{-1}\right)$.

Intracellular ATP measurements. After seeding overnight, the cells were treated as indicated. Intracellular ATP levels were determined in cell lysates using a luciferase-based assay as per the manufacturer's instructions (Sigma-Aldrich). Results were normalised to the total protein level in cell lysate, as determined by the Bradford method (Bio-Rad) (Cheng et al, 2012, 2013).

Extracellular flux assay. To determine the mitochondrial and glycolytic function in pancreatic cancer cells, we used the bioenergetic function assay (Nicholls et al, 2010; Dranka et al, 2012) using Seahorse XF96 Extracellular Flux Analyzer (Seahorse Bioscience, North Billerica, MA, USA). After seeding overnight, the cells were washed with unbuffered assay media (DMEM, CellGro, Manassas, VA, USA; cat. no. 90-113-PB, supplemented with $5.5 \mathrm{~mm}$ glucose and $4 \mathrm{~mm}$ L-glutamine, but no pyruvate and no sodium bicarbonate were added) and conditioned for $1 \mathrm{~h}$ before measurements. Eight baseline oxygen consumption rate (OCR) and extracellular acidification rate (ECAR) measurements over $1 \mathrm{~h}$ were recorded after cell conditioning in the assay medium, and the values were averaged from the last six (most stable) readings. ECAR expressed as $\mathrm{mpH} \mathrm{min}^{-1}$ was automatically converted to proton production rate (PPR) expressed as $\mathrm{pmol} \mathrm{H}^{+} \min ^{-1}$ using the determined buffer capacity of the media and the chamber volume in XF96 Analyzer. The PPR-dependent changes are very similar to ECAR; however, we chose PPR instead of ECAR due to the ease of normalisation, and to the logarithmic nature of ECAR with regard to the rate of production of $\mathrm{H}^{+}$. OCR and ECAR (or PPR) values have been used as measures of mitochondrial respiration and glycolytic activity, respectively. Differences in mitochondrial respiration and/or acid extrusion (i.e., basal glycolysis status) are indicative of their sensitivity to glycolytic inhibitors.

Glucose consumption measurement. After seeding overnight, the cells were treated with a fresh medium as indicated. The glucose levels in the medium were determined using an enzyme-based assay (Sigma-Aldrich, GAHK-20) as per the manufacturer's instructions with a slight modification. Briefly, the assay is based on hexokinase-mediated phosphorylation of glucose to glucose-6phosphate $(\mathrm{G} 6 \mathrm{P})$ with subsequent reduction of $\mathrm{NAD}^{+}$to $\mathrm{NADH}$ by G6P catalysed by G6P dehydrogenase. The total amount of $\mathrm{NADH}$ formed, as measured by spectrophotometry, is calibrated against the concentration of glucose standard (see Supplementary Figure 1) and used for glucose quantification. An incubation time of $1 \mathrm{~h}$ was chosen rather than manufacturer-recommended $15 \mathrm{~min}$, as 2-DG slightly decreased the rate of product (NADH) formation, but did not affect the final (total) amount of the product detected (Supplementary Figure 1).

Glutamine measurement. The glutamine levels in the media were determined by using an enzyme-based assay (Sigma-Aldrich, GLN1) as reported earlier (Kung et al, 2011; Mullen et al, 2012). Briefly, L-glutamine is deaminated with glutaminase, to form L-glutamate, which in a subsequent reaction reduces $\mathrm{NAD}^{+}$to $\mathrm{NADH}$ in the presence of L-glutamate dehydrogenase. The total amount of NADH reflects the sum of both glutamine and glutamate. To determine the concentration of glutamine, the amount of $\mathrm{NADH}$ formed in the presence and absence of glutaminase is measured and the amount of glutamine calculated after determining the contribution of glutamate. The accuracy of the glutamine assay used was confirmed by validation against LC-MS-based glutamine quantification.

Clonogenic assay. The cells were seeded as indicated in six-well plates and treated with 2-DG. The plates were left within the incubator and media changed with vehicle or 2-DG every 3-4 days until cells in the control wells formed sufficiently large clones. The cell survival fractions were calculated as described previously (Cheng et al, 2012, 2013).

Cell proliferation assay. The cell proliferation was measured using a label-free, non-invasive cellular confluence assay by IncuCyte Live-Cell Imaging Systems (Essen Bioscience, Ann Arbor, MI, USA). MiaPaCa-2 (1000 cells/well) and Capan-2 (3000 cells/well) were seeded overnight on a 96 -well plate, placed in an XL-3 incubation chamber maintained at $37^{\circ} \mathrm{C}$ and the cells were photographed using a $\times 10$ objective. This system enables collection of live cell images at 2 -h intervals over several days. 
Cell confluence was calculated using IncuCyte 2011 software. The IncuCyte Analyzer provides real-time cellular confluence data based on segmentation of high-definition phase-contrast images. The cell proliferation is expressed as an increase in percentage of confluence.

Statistics. All results are expressed as the mean \pm s.d. Comparisons among groups of data were made using a one-way ANOVA with Tukey's post hoc analysis. A $P$-value $<0.05$ was considered to indicate statistical significance. The synergistic effects of drug combinations were validated by the combination index (CI) method (Chou and Talalay, 1981) for multidrug treatment using the CalcuSyn software (Bosoft, Cambridge, UK). The following CI criteria were used: $\mathrm{CI}>1$ indicates antagonism; $\mathrm{CI}=1$ indicates additivity; $1>\mathrm{CI}>0.7,0.7>\mathrm{CI}>0.3$ and $0.3>\mathrm{CI}>0.1$ indicate slight synergy, synergy and strong synergy, respectively.

\section{RESULTS}

The effect of 2-DG on intracellular ATP and colony formation in pancreatic adenocarcinoma cell lines. We investigated the effect of 2-DG on intracellular ATP levels in six different pancreatic cancer cells of different genetic backgrounds (Figure 1A). These cells are differentially sensitive to 2-DG with respect to the extent of ATP depletion, with MiaPaCa-2 and Capan-2 being the most and least sensitive cell lines, respectively. Figure 1B shows a heat map representation of intracellular ATP levels in MiaPaCa-2 and Capan-2 cells (coloured areas from brown to blue indicate a progressive decrease in ATP from 100\% to 30\%) in response to different concentration and time of treatment with 2-DG. As shown, 2-DG treatment induced ATP depletion in MiaPaCa- 2 cells in a time- and concentration-dependent manner, whereas Capan-2 cells were exceedingly resistant to 2-DG under these conditions (Figure 1B). Similar heat maps of ATP depletion in response to 2-DG in other pancreatic cells are shown in the Appendix (Supplementary Figure 2). Intracellular ATP levels (calculated after normalisation to total protein) are shown in Supplementary Tables $1-4$. Results indicate that different cell lines tested exhibit different sensitivity to 2-DG.

Next, we used the clonogenic assay (measuring of the ability of cells to form colonies) to monitor the anti-proliferative effects of 2-DG. Figure 1C shows the clonogenic results obtained in these cells, indicating that $2-\mathrm{DG}$ caused a vastly decreased colony formation in MiaPaCa-2 cells exhibiting greater sensitivity as compared with Capan-2 cells. This is consistent with the ATP depletion results (Figure 2A and B). As shown in Figure 1D, the calculated cell survival fraction in the presence of 2-DG was the lowest in AsPC-1 and MiaPaCa-2 cells, while Capan-2 cells were resistant to the anti-glycolytic drug.

Mapping of bioenergetics in pancreatic cancer cells. To better understand the mechanism of the differential sensitivity of pancreatic ductal adenocarcinoma cells to 2-DG, we investigated in detail their metabolic and energetic requirements. Pancreatic cancer cells with different genetic backgrounds were analysed in real-time using a Seahorse Extracellular Flux (XF) 96-well Analyzer. Both cellular OCR, resulting from oxidative phosphorylation, and ECAR, associated with glycolytic metabolism, were simultaneously monitored in six pancreatic cancer cell lines (Figure 2A). The ECAR values have been converted to proton production rates (PPR) in order to directly reflect the efflux of lactic acid. Figure 2B shows the relative two-dimensional OCR/ PPR bioenergetic map calculated from the slopes in oxygen $\left(\Delta \mathrm{O}_{2}\right)$ and proton $\left(\Delta \mathrm{H}^{+}\right)$traces shown in Figure 2A. Results indicate that the cell line Capan-2 is the least glycolytic, whereas MiaPaCa-2 is the most glycolytic. There is more than a five-fold difference in the PPR parameters as compared with only a two-fold change in the
OCR value over the six cell lines tested. The two cell lines, Capan-2 and MiaPaCa-2, were particularly interesting because the basal OCR values are nearly identical in both cells, although the PPRs, the surrogate marker of glycolysis, differ by a factor of five.

Complementary to measurements of extracellular acidification rates, the utilisation of glucose in the cell culture media was also monitored with time to directly measure the rate of glycolysis. Figure $2 \mathrm{C}$ shows the changes in the concentration of glucose in media as a function of incubation time for six different cell lines tested. A very good correlation between PPR and glucose consumption data was observed (Figure 2D), further corroborating the utility of PPR as a potential marker of glycolysis. The ratio of PPR values to the rates of glucose consumption is not constant across different cell lines indicating different pathways/profiles of glucose utilisation in different pancreatic cancer cells tested in this study. Similarly, despite five-fold differences in the PPR values between MiaPaCa-2 and Capan-2 cells, the intracellular level of ATP is not very different in both cell lines (Supplementary Table 1), indicating the different profiles of ATP synthesis and/or different rates of ATP utilisation.

Effect of inhibition of glycolysis on intracellular ATP level and cell proliferation in pancreatic cancer cells of different basal glycolytic status. We investigated in detail the relationship between the basal rates of glycolysis and the extent of depletion of intracellular ATP in the pancreatic cancer cells in response to 2-DG treatment. Figure 3A shows a close correlation between the basal rate of proton production (also extracellular acidification) and intracellular ATP depletion by 2-DG. Similarly, there was a very good correlation between intracellular ATP loss treated with 2-DG and the basal glucose consumption rates (Supplementary Figure 3A). A good correlation between basal glycolytic status, as measured by PPR or rates of glucose consumption, and the extent of inhibition of colony-forming ability in these cells was also observed (Figure $3 \mathrm{~B}$ and Supplementary Figure 3B). These results suggest that monitoring changes in bioenergetics in pancreatic cancer cells may help select appropriate metabolism-based drug therapy for inhibiting pancreatic cancer cell proliferation.

To determine whether the effects observed in response to 2-DG are due to differences in the glycolytic inhibitory effects in the six pancreatic cancer cells, we continuously monitored (for $6 \mathrm{~h}$ ) the effect of 2-DG on the proton production and glucose consumption rates. Results show that 2-DG dose-dependently decreased ECAR values and glucose consumption to the same extent in different cells (Figure 4A and B). Thus, the observed differences in sensitivity to inhibition of glycolysis, as determined by monitoring intracellular ATP levels and colony formation in these cells, are not due to the differential effects of 2-DG on glycolytic pathway. Therefore, we also investigated the effect of 2-DG on glutamine uptake under the same conditions (Figure 5A, bottom). Results indicate that there are no remarkable differences in glutamine consumption in 2-DG-treated pancreatic cells.

Dual targeting of mitochondrial and glycolytic pathways of ATP production in pancreatic cancer cells: the synergistic effect of metformin and 2-DG. It has been reported that dual targeting of cancer cells with mitochondrial and glycolytic inhibitors synergistically inhibits tumour cell proliferation (Liu et al, 2001; Maschek et al, 2004; Cheng et al, 2012, 2013). We tested the combined effect of metformin, a relatively safe anti-diabetic drug that has recently been shown to inhibit pancreatic cancer in diabetics, and 2-DG. Metformin is also a weak inhibitor of mitochondrial metabolism (Supplementary Figure 4). Figure 5A, top panel shows the intracellular ATP levels in all of these cells treated with metformin and 2-DG, alone and in combination, for $24 \mathrm{~h}$. The combination of 2-DG and metformin caused the maximal depletion of ATP levels, even in cell lines resistant to 2-DG or metformin monotherapy. 


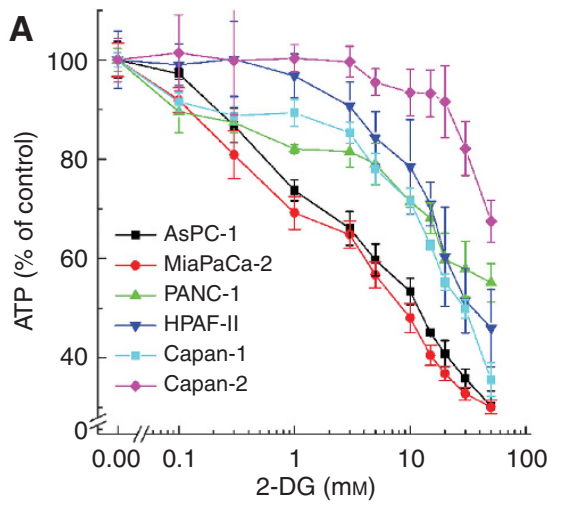

C

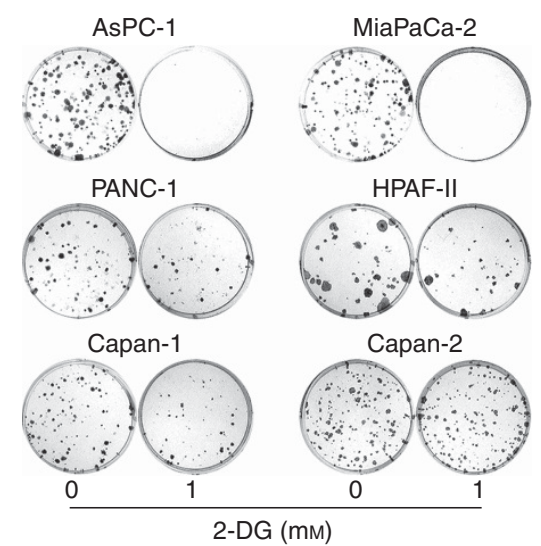

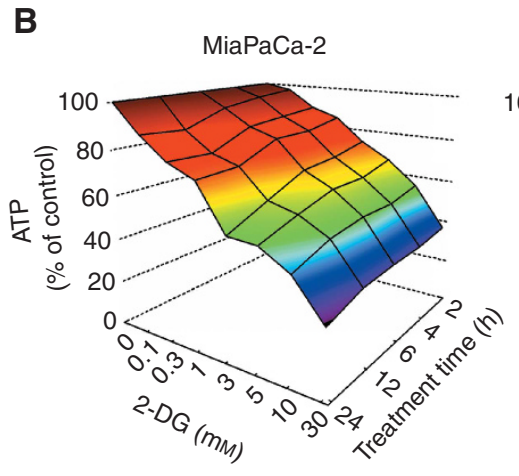
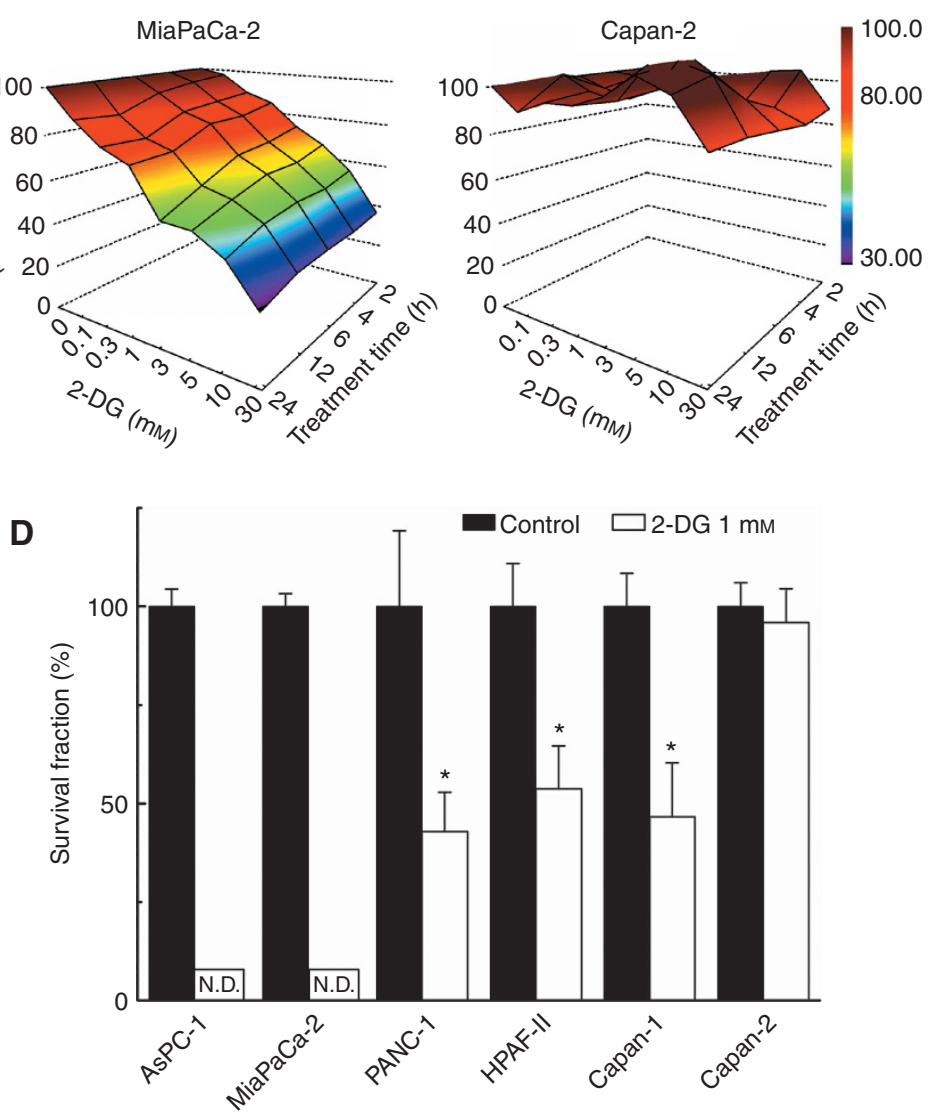

Figure 1. Susceptibility of pancreatic cancer cells to 2-DG. (A) Six pancreatic cancer cells were treated with 2-DG (0.1-50 mm) for $6 \mathrm{~h}$ and intracellular ATP levels were measured using a luciferase-based assay. Data are shown as a percentage of control (non-treated) cells after normalisation to total cellular protein in each well. (B) A three-dimensional representation showing the concentration and time-dependent effects of 2-DG on intracellular ATP levels in MiaPaCa-2 cells (left) and in Capan-2 cells (right). (C) The effect of 2-DG on colony formation in pancreatic cancer cells treated with 2-DG (1 mM). (D) The survival fractions of six pancreatic cancer cell lines after treatment with 2-DG.

$\mathrm{ND}=$ colonies not detected.

Metformin treatment alone slightly increased glucose consumption in most of the pancreatic cancer cell lines tested (Figure 5A, middle), attributable to a compensatory response to inhibition of mitochondrial respiration. This response was blocked when metformin was combined with 2-DG. However, it was recently reported that metformin impairs glucose metabolism in Caco-2 cells as a result of binding to hexokinase enzyme responsible for glucose phosphorylation (Salani et al, 2013). We monitored the activity of hexokinase in cell-free system using an enzyme-based assay in a 96-well plate in response to 2-DG or metformin at several concentrations (Supplementary Figure 5). 2-Deoxyglucose competes with glucose for phosphorylation by hexokinase and as shown in Supplementary Figure 5 top panel, there was a dosedependent decrease in the rate of hexokinase-dependent phosphorylation of glucose. In contrast, metformin had no effect on hexokinase-mediated phosphorylation of glucose (Supplementary Figure 5, bottom). In most of the PDAC cells examined, the combined use of 2-DG and metformin synergistically enhanced intracellular ATP depletion. Results show that even in poorly glycolytic pancreatic cells, Capan-1 and 2 (Figure 5A, top), 2-DG and metformin synergistically enhanced depletion of intracellular ATP. Supplementary Figure 6 shows the heat maps of ATP depletion in response to 2-DG and metformin treatment of MiaPaCa-2 cells (Supplementary Figure 6A after a 6-h treatment and Supplementary Figure 6C after a 24 -h treatment). Calculation of the CI shows a strong synergy for the combined treatment of 2-DG and metformin $(\mathrm{CI}<0.3)$ in depleting intracellular ATP (Supplementary Figure 6B and D). To determine the mitochondrial inhibitory effects of metformin in these cells, we measured OCR in the Seahorse apparatus using cells treated with different concentrations of metformin (Supplementary Figure 4). Metformin dose-dependently inhibited OCR in these cells that is attributed to its known inhibitory effect on mitochondrial complex 1 (Zannella et al, 2013). However, results indicate that metformin inhibits OCR to the same extent in all pancreatic cancer cells. We then investigated the effects of 2-DG and metformin on glutamine utilisation in the six cell lines (Figure 5A, bottom). Metformin alone or together with 2-DG inhibited glutamine consumption in each of the six pancreatic cancer cell lines, which we attribute to a decreased glutamine demand due to inhibited cellular metabolism and proliferation. Taken together, dual targeting of mitochondrial and glycolytic bioenergetics with 2-DG and metformin could significantly slow down the metabolism of pancreatic cancer cells, regardless of their glycolytic status (e.g., MiaPaCa-2, highly glycolytic and Capan-2, poorly glycolytic cancer cells).

We measured cell proliferation using the IncuCyte Analyzer. Figure 5B and Supplementary Figure 7 show the cell proliferation curves as measured by cell confluence kinetics for MiaPaCa-2 and Capan-2 cells treated with metformin and 2-DG alone and in combination. The actual phase-contrast images recorded are shown in Supplementary Figure 8 . These results clearly indicate that metformin and 2-DG synergistically mitigate pancreatic cancer cell proliferation. The results obtained for 2-DG alone (Supplementary Figure 7) reiterate the sensitivity of MiaPaCa-2, but not Capan-2, to glycolytic inhibition. 

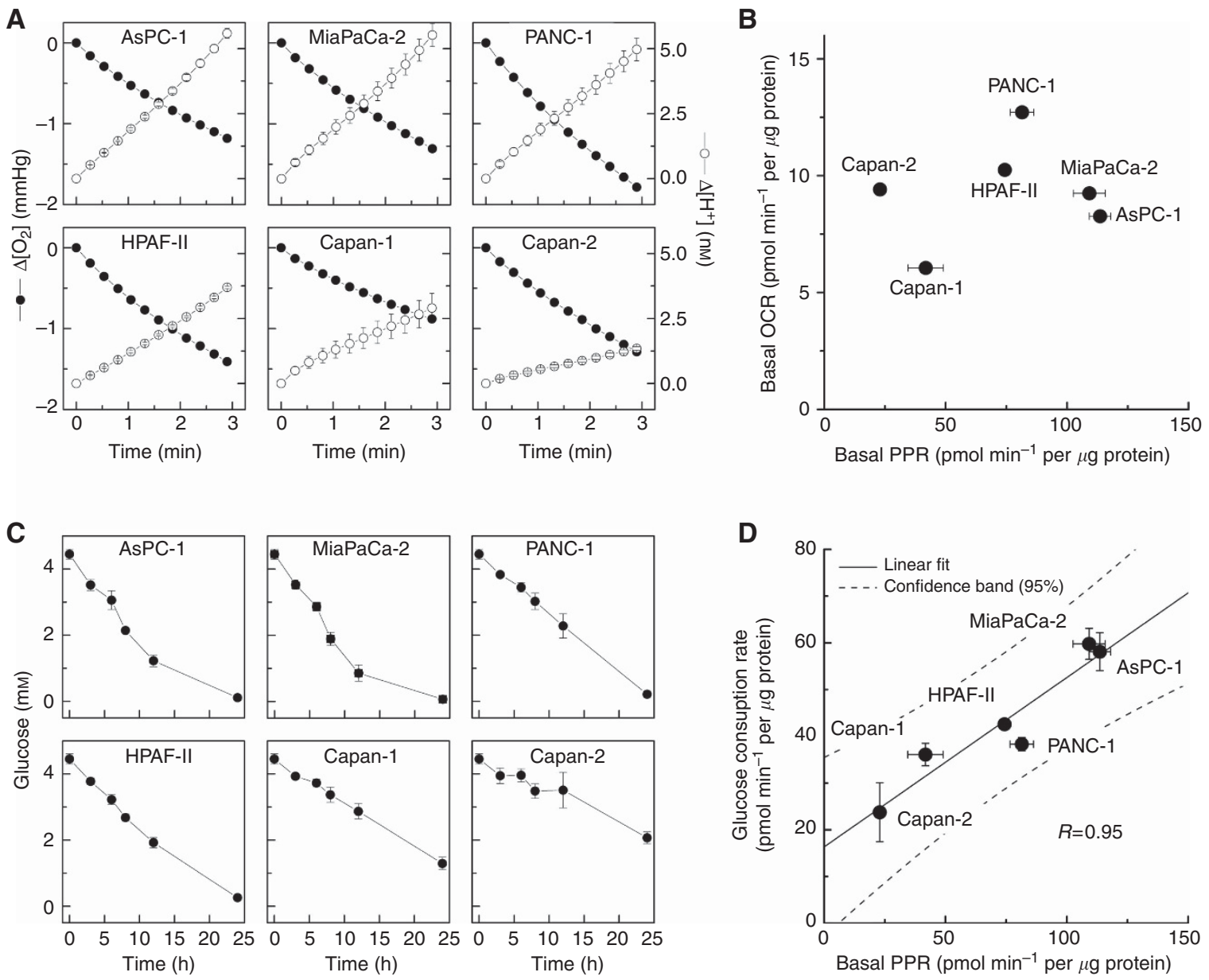

Figure 2. Profiling of bioenergetics of pancreatic cancer cells. (A) Oxygen consumption $\left(\Delta \mathrm{O}_{2}\right)$ and proton production $\left(\Delta \mathrm{H}^{+}\right)$traces in six pancreatic cancer cell lines as monitored with a Seahorse XF96 Analyzer, as described in Materials and Methods section. The changes in $\mathrm{O}_{2}$ and $\mathrm{H}^{+}$concentrations were normalised to $1 \mu \mathrm{g}$ of cellular protein. (B) Two-dimensional map of oxygen consumption rate (OCR) and proton production rate (PPR) measured in six pancreatic cancer cell lines. (C) Changes in glucose concentration in media incubated with six pancreatic cancer cell lines. (D) Relationship between the basal PPR values and glucose consumption rates. The glucose consumption rate ( $0-12 \mathrm{~h}$, calculated from $(C)$ is plotted against the basal PPR value of each cell line. Values are mean \pm s.d. $(n=4-6)$.

The inhibition of glycolysis with 2-DG in MiaPaCa-2 cells treated with conventional chemotherapeutic drugs (gemcitabine or doxorubicin) synergistically inhibited cell proliferation (Supplementary Figure 9A and B). As shown, 2-DG (0.5 mM) greatly augmented the inhibitory effect of gemcitabine $(10 \mathrm{~nm})$ or doxorubicin (10 nM).

We noted an inverse relationship between the reported levels of cyclooxygenase-2 (COX-2) enzyme (Deer et al, 2010) and PPR, a surrogate marker of glycolysis, in various PDACs tested (Supplementary Figure 10A). As metformin was shown to inhibit COX-2 (Kalariya et al, 2012; Kim and Choi, 2012), we have also tested whether COX-2 inhibitor, celecoxib, would sensitise the Capan-2 cells to 2-DG. As shown in Supplementary Figure 10B and $\mathrm{C}$, combination of celecoxib and 2-DG induced synergetic depletion of total intracellular ATP in Capan-2. Similar effects were also observed in MiaPaCa-2 cells (data not shown).

\section{DISCUSSION}

In this study, we show for the first time as to how two-dimensional mapping of bioenergetic profiles in multiple pancreatic cancer cell lines of different genetic background can be used to provide new therapeutic insight for inhibiting pancreatic tumour cell proliferation. Highly glycolytic cells (e.g., MiaPaCa-2) are exquisitely sensitive to glycolytic inhibition using 2-DG, whereas the poorly glycolytic cells (e.g., Capan-2) are relatively insensitive to 2-DG treatment. However, dual targeting of mitochondrial and glycolytic bioenergetics, using metformin and 2-DG, is effective in inhibiting proliferation of different pancreatic cancer cell phenotypes. The present results suggest that the efficacy of metformin monotherapy or anti-glycolytic monotherapy can be synergistically enhanced when used together.

Tumour cells adapt to various microenvironments and energyrelated challenges by switching between glycolysis, glutaminolysis, and mitochondrial metabolism (Daye and Wellen, 2012; Ferreira et al, 2012). The metabolic reprogramming provides the cellular energy required for increased cancer growth and proliferation (Jones and Schulze, 2012). Oncogenes actively reprogram tumour cell metabolism. Cellular signalling and metabolism in tumour cells are interconnected (Jones and Schulze, 2012; Regel et al, 2012). Many core oncologic signal pathways (K-Ras, cMYC, p53, and LLKB1) affect cell proliferation and cell metabolism in pancreatic carcinogenesis (Le et al, 2012). Increased aerobic glycolysis and glutaminolysis are characteristic phenotypic traits in tumours (Kurtoglu and Lampidis, 2009; Son et al, 2013). Recent studies have shown a link between glutamine metabolism and K-Ras pathways 

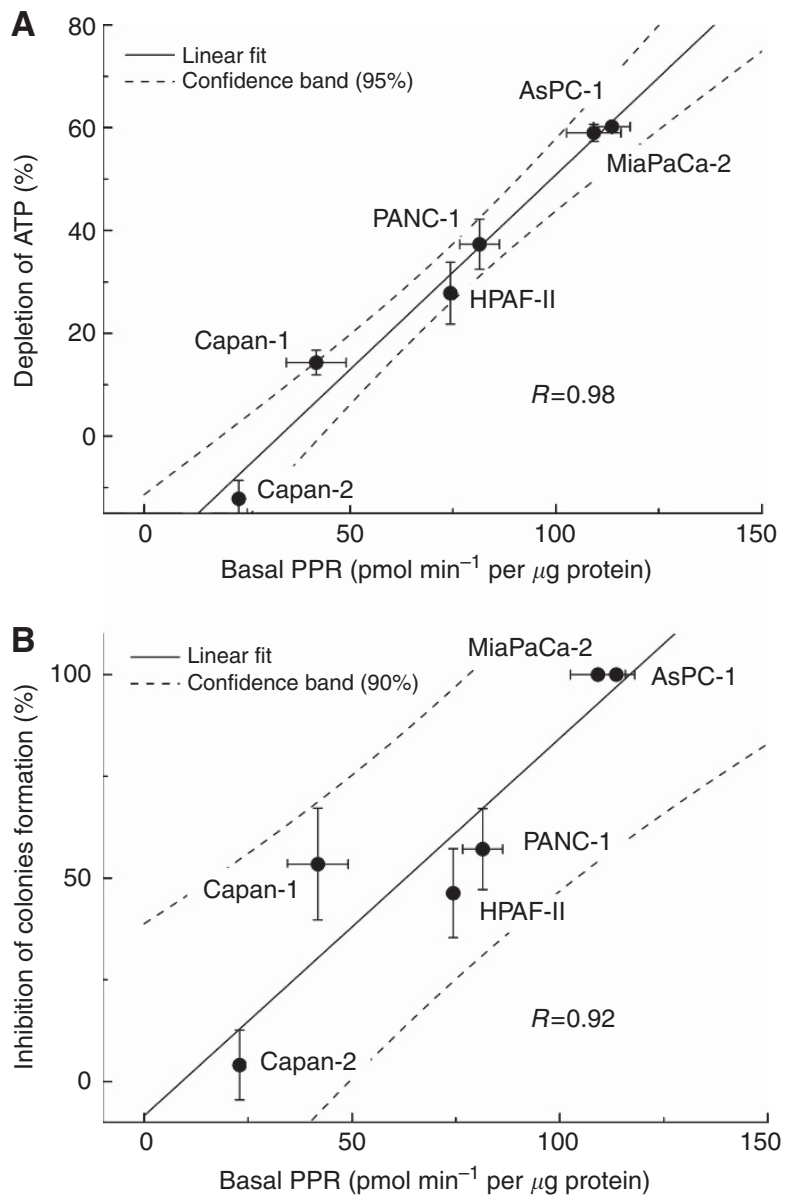

Figure 3. Relationship between ATP depletion, inhibition of colony formation and basal PPR values of pancreatic cancer cells in response to 2-DG treatment. (A) Pancreatic cancer cells were treated with 2-DG $(3 \mathrm{~mm})$ for $24 \mathrm{~h}$ and the extent of ATP depletion was plotted against the PPR values. Negative value for Capan-2 corresponds to a small increase in ATP level after treatment with 2-DG. (B) Graph showing the relationship between the extent of inhibition of colony formation by 2-DG (1 mM) and determined basal PPR values for each cell line.

in pancreatic cancer cell maintenance and growth (Wenner, 2012). Under conditions of hypoxia or energy stress, such as enhanced glycolysis and/or inhibition of mitochondria, glutamine provides an alternate viable amino-acid source.

Targeting the mitochondrial bioenergetics and the glycolysis pathway in tumour cells has been an attractive chemotherapeutic strategy for many years (Pathania et al, 2009). This strategy was shown to be effective even in slow-growing hypoxic tumour cells normally resistant to conventional chemotherapeutic agents. Early on, it was reported that rhodamine-123, a lipophilic cationic fluorescent dye, selectively accumulated into tumour cells, inhibiting mitochondrial respiration (Bernal et al, 1983). Tumour cells became hypersensitised to inhibitors of glycolysis such as 2-DG (Liu et al, 2001). 2-Deoxyglucose inhibits glycolysis by being phosphorylated by hexokinase and converted to a nonmetabolisable, 2-deoxyglucose-6-phosphate (Pelicano et al, 2006; Dwarakanath and Jain, 2009). Previously, we reported that combining mitochondria-targeted cationic agents (Mito-CP, Mito-chromanol, and Mito-Q) with 2-DG is a better strategy to selectively inhibit breast cancer cell proliferation (Cheng et al, 2012, 2013). Combined administration of Mito-CP and 2-DG also caused tumour regression in a breast cancer xenograft model
(Cheng et al, 2012). In this work, the combined treatment with metformin and 2-DG caused nearly 60\% ATP depletion and almost complete inhibition of cell proliferation even in Capan-2 cells, which are the most resistant to 2-DG. On the basis of the published data on the selective anticancer effects of combination of mitochondria-targeted agents with 2-DG (Cheng et al, 2012, 2013), we expect that combination of metformin with 2-DG should also selectively target cancer cells, which is supported by recent reports (Ben Sahra et al, 2010; Cheong et al, 2011). Although the present data were obtained from in vitro cell culture experiments (without taking into account the tumour microenvironment), we envision that the proposed strategy should be translatable to in vivo pancreatic cancer xenografts. In fact, the combination of metformin and 2-DG was recently shown to be effective in in vivo models, such as prostate and breast cancer xenografts (Ben Sahra et al, 2010; Cheong et al, 2011).

Metformin exists as a weak hydrophilic cation at physiological $\mathrm{pH}$ and targets mitochondria, although not very efficiently (Zannella et al, 2013). It is not clear how metformin is transported into cancer cells. In hepatocytes, metformin uptake is facilitated by an organic cation transporter 1 (Wang et al, 2002; Segal et al, 2011). Thus, the concentration of metformin required to inhibit complex 1 in the mitochondrial electron transport chain is much higher compared with the strongly cationic agents conjugated to the triphenylphosphonium cation (Cheng et al, 2012). Nevertheless, it is likely that the metformin-treated cells became hypersensitised to glycolytic inhibition by 2-DG. Metformin has been shown to synergise with 2-DG in various cancer cell lines, but pancreatic cancer cells were not tested (Ben Sahra et al, 2010; Cheong et al, 2011). In the present work, we show that metformin/2-DG combined therapy was also effective in pancreatic cancer cells that are resistant to anti-glycolytic monotherapy. Recently, it has been postulated that slowing down glycolysis via inhibition of hexokinase activity may contribute to the anti-proliferative effects of metformin (Salani et al, 2013). We did not, however, observe a similar phenomenon in any of the pancreatic cancer cell lines tested, in agreement with the reported data for prostate cancer cells (Ben Sahra et al, 2010). There is a renewed interest in more potent metformin analogues (e.g., phenformin) in pancreatic cancer treatment, despite the fact that this drug was withdrawn from the market for treatment of diabetes due to excess lactic acidosis. More recent data suggest that metformin treatment increased the dependency of prostate cancer cells on reductive glutamine metabolism (Fendt et al, 2013). Prostate cancer cells are dependent on fatty-acid oxidative metabolism for ATP synthesis. Glutamine metabolism contributes to de novo lipid synthesis through reductive carboxylation (Metallo et al, 2012). It was suggested that metformin combined with inhibitors of glutamine metabolism might be synergistically beneficial in prostate cancer treatment (Fendt et al, 2013). However, metformin treatment diminished pancreatic cancer cell proliferation and as a consequence 'slowed down' glutamine utilisation. Thus, it is conceivable that metformin-mediated changes in bioenergetics are dependent on the cancer cell type and their intrinsic metabolic requirements.

In the tumour microenvironment, glucose levels are considerably lower due to increased consumption through glycolysis. Under conditions that are unique to tumour microenvironments, metformin's in vivo anti-tumour efficacy or metformin-induced chemical synthetic lethality (Menendez et al, 2012) can be significantly higher. Targeting glycolysis and related cellular energy pathways, alone and in combination, may provide an effective and viable therapeutic option in pancreatic cancer treatment (Pelicano et al, 2006; Dwarakanath and Jain, 2009).

Although the differences in glycolytic state and response to drug treatment may be related to differentiated expression of 
A

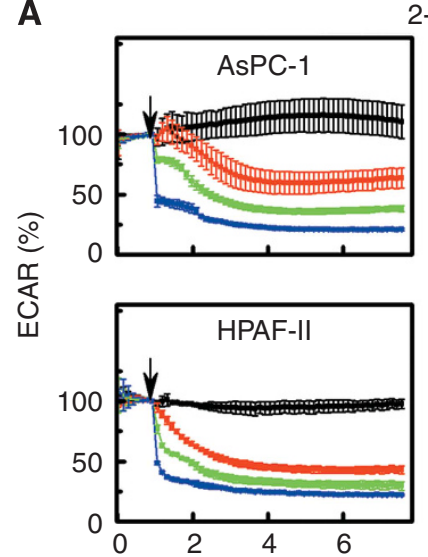

2-DG (mM): $\rightarrow 0 \rightarrow 1 \rightarrow$
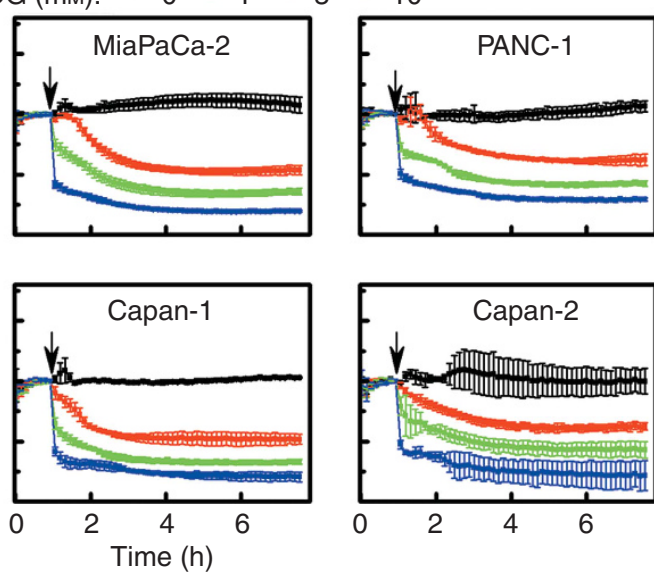

B

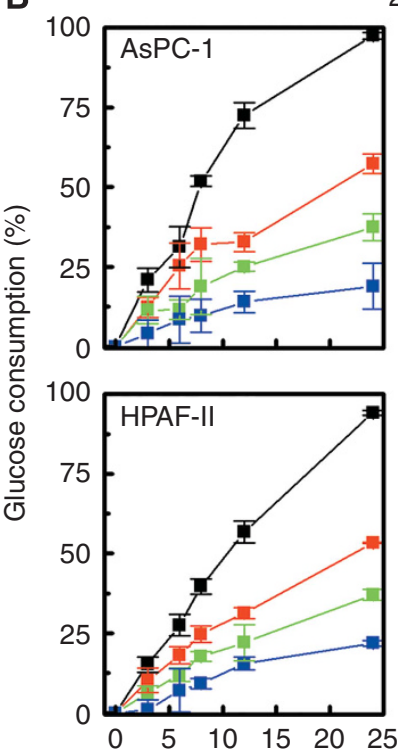

2-DG (mM): $-0-0-1-1-3-10$
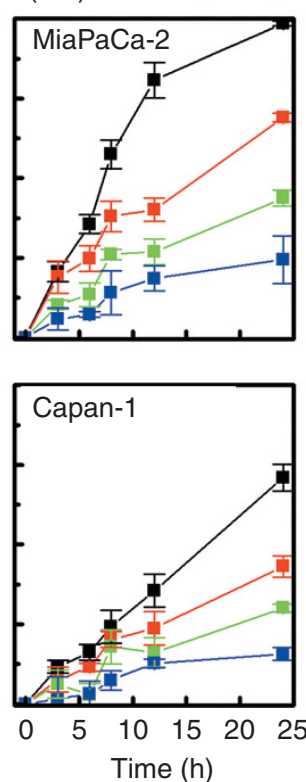
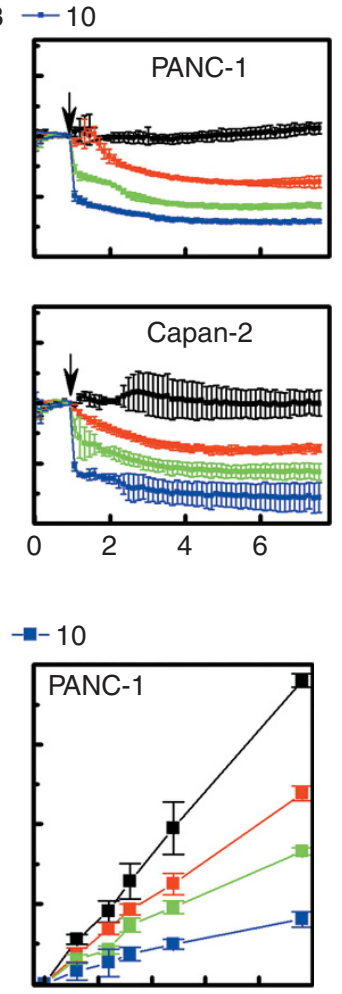

10

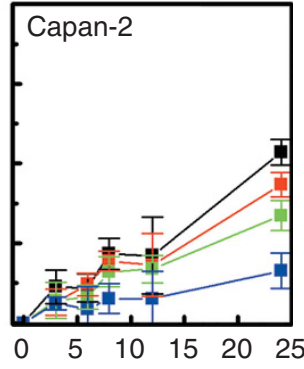

Figure 4. Real-time monitoring of changes in ECAR and glucose consumption in pancreatic cancer cells treated with 2-DG. (A) Pancreatic cancer cells were treated with different concentrations of 2-DG and the ECAR values measured with a Seahorse XF96 Flux Analyzer. The arrows indicate the time point of 2-DG injection. The ECAR value recorded immediately before injection of 2-DG is 100\%. (B) The kinetics of glucose consumption by pancreatic cells at different concentrations of 2-DG. The glucose levels in the media were measured in response to 2-DG treatment under conditions similar to those of $(\mathbf{A})$ and presented as the amount consumed in relation to the initial level in the medium.

Glut transporter (Maher et al, 2005), we sought alternate explanations for this effect. During the process, we discovered a correlation between the glycolytic rates and the reported relative protein expression levels (reported in Deer et al, 2010) of the COX-2 enzyme in the PDACs tested in this study. As shown in Supplementary Figure $10 \mathrm{~A}$, there is an inverse relationship between COX-2 protein expression and the basal PPR (glycolytic marker) levels. This study also showed that the COX-2 protein expression corresponds to the function of COX-2 in these cells (Deer et al, 2010).The COX-2 enzyme, typically overexpressed in pancreatic cancer (Tucker et al, 1999), has been causally linked to resistance to chemotherapeutic drugs. Thus, specific inhibitors of COX-2, celecoxib, for example, may potentiate the growth inhibition of 2-DG. Metformin has also been shown to suppress the expression and activity of COX-2 (Kim and Choi, 2012). We reasoned that this mechanism (COX-2 inhibition) is likely, at least partially, responsible for the growth inhibitory effect of 2-DG in Capan-2 cells. In support of this hypothesis, we observed that the combined treatment of celecoxib and 2-DG synergistically enhanced the depletion of cellular ATP (Supplementary Figure 10B). Although the exact mechanism for the synergistic ATP-depleting effect of celecoxib and 2-DG (Supplementary Figure 10C) remains to be determined, this is a new and intriguing finding with potential clinical and translational significance based on bioenergetic mapping.

Although the exact mechanism for the synergistic effects of 2-DG and gemcitabine, doxorubicin and celecoxib remains to be determined, these findings open up a new therapeutic strategy that combines glycolytic inhibitors with standard-of-care drugs in pancreatic cancer treatment.

In summary, we have shown in this study that 2-DG is very effective in depleting intracellular ATP and inhibiting proliferation in highly glycolytic cells but not in poorly glycolytic cells. However, metformin, a weak inhibitor of mitochondrial metabolism, and 2-DG are able to synergistically enhance ATP depletion and inhibit proliferation in pancreatic cancer cells, including the 2-DG-resistant cell lines. Bioenergetic mapping of pancreatic cancer cells using the Seahorse Analyzer may provide new insight into how targeted blockades of glycolytic, 

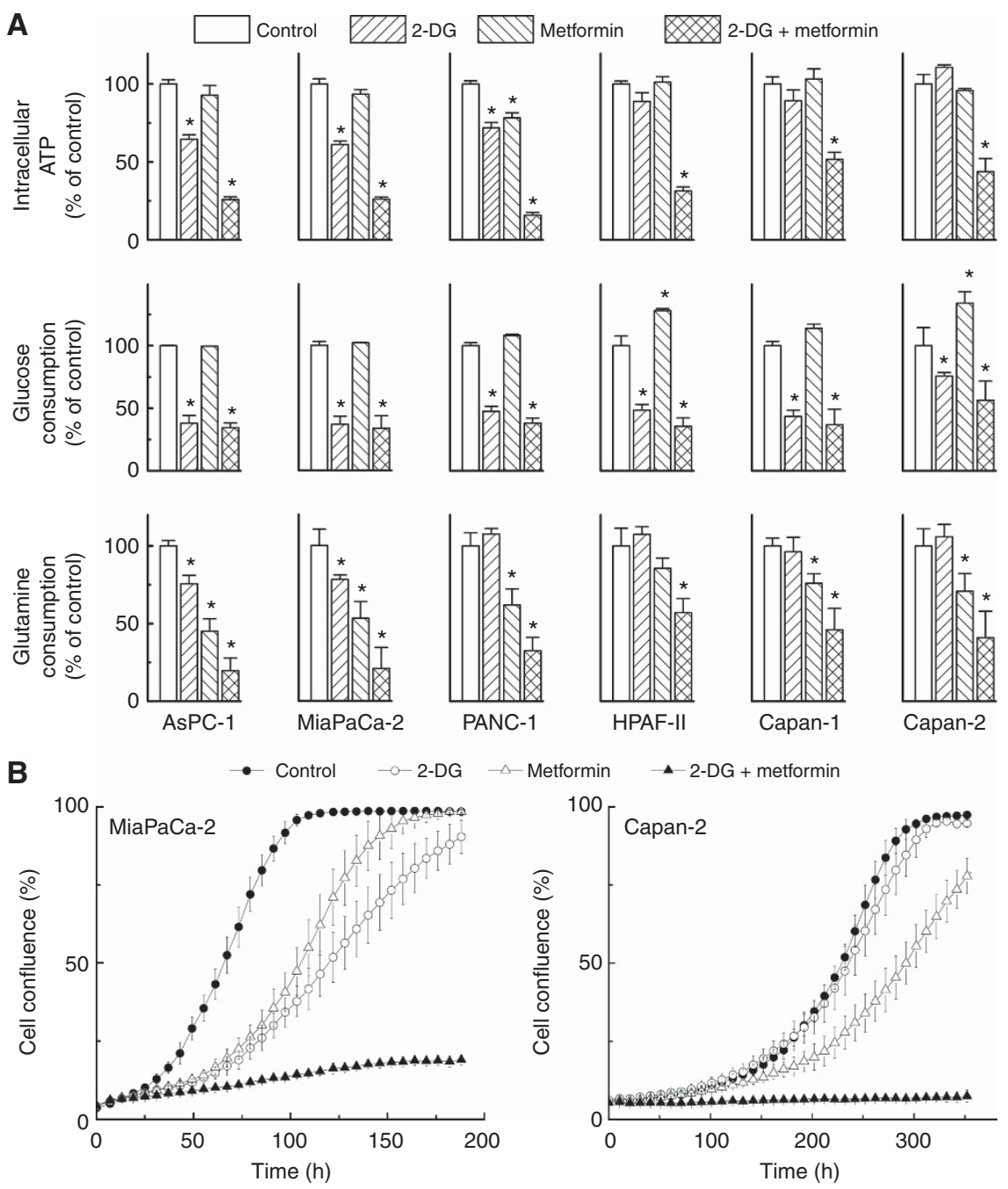

Figure 5. Synergistic depletion of ATP and inhibition of cell proliferation by 2-DG and metformin. (A) Pancreatic cancer cells were treated with 2-DG (3 mm) or metformin ( $10 \mathrm{~mm}$ ) independently and together for $24 \mathrm{~h}$ and intracellular ATP levels (top), glucose consumption (middle), and glutamine consumption (bottom) were determined, normalised to total cellular protein amount and expressed as percentage of untreated cells. Data shown represent the mean \pm s.d. ${ }^{\star} P<0.05$, $(n=4)$ vs control in each cell line. (B) Effects of 2-DG and metformin alone and together, on cell proliferation. MiaPaCa-2 and Capan-2 cells were treated with 2-DG (0.5 mm in MiaPaCa-2, $1 \mathrm{~mm}$ in Capan-2 cells) or metformin (1 mM) alone and together. Cell proliferation was monitored in real time with the continuous presence of indicated treatments until the end of each experiment. The changes in cell confluence are used as a surrogate marker of cell proliferation. Data shown are the mean \pm s.d. $(n=6)$.

glutaminolytic, and mitochondrial metabolism pathways can be effectively used in cancer treatment. We hope that the bioenergetic profiling approach will enable the selection and use of metabolic inhibitors in combination with the standard-ofcare drugs in pancreatic cancer treatment.

\section{ACKNOWLEDGEMENTS}

This study was supported by the National Institutes of Health (grant number R01CA152810 to BK) and a grant from the MCW Cancer Center (to MBD). We also acknowledge the use of the mass spectrometer in the MCW Cancer Center Bioenergetic Shared Resource.

\section{REFERENCES}

American Cancer Society (2013) Cancer Facts \& Figures 2013. pp 25-34. American Cancer Society: Atlanta, GA, USA.
Ben Sahra I, Laurent K, Giuliano S, Larbret F, Ponzio G, Gounon P, Le Marchand-Brustel Y, Giorgetti-Peraldi S, Cormont M, Bertolotto C, Deckert M, Auberger P, Tanti JF, Bost F (2010) Targeting cancer cell metabolism: the combination of metformin and 2-deoxyglucose induces p53-dependent apoptosis in prostate cancer cells. Cancer Res 70: $2465-2475$.

Bernal SD, Lampidis TJ, McIsaac RM, Chen LB (1983) Anticarcinoma activity in vivo of rhodamine 123, a mitochondrial-specific dye. Science 222: 169-172.

Cheng G, Zielonka J, Dranka BP, McAllister D, Mackinnon Jr AC, Joseph J, Kalyanaraman B (2012) Mitochondria-targeted drugs synergize with 2-deoxyglucose to trigger breast cancer cell death. Cancer Res 72: 2634-2644.

Cheng G, Zielonka J, McAllister DM, Mackinnon Jr AC, Joseph J, Dwinell MB, Kalyanaraman B (2013) Mitochondria-targeted vitamin E analogs inhibit breast cancer cell energy metabolism and promote cell death. BMC Cancer 13: 285.

Cheong JH, Park ES, Liang J, Dennison JB, Tsavachidou D, Nguyen-Charles C, Wa CK, Hall H, Zhang D, Lu Y, Ravoori M, Kundra V, Ajani J, Lee JS, Ki HW, Mills GB (2011) Dual inhibition of tumor energy pathway by 2-deoxyglucose and metformin is effective against a broad spectrum of preclinical cancer models. Mol Cancer Ther 10: 2350-2362. 
Chou TC, Talalay P (1981) Generalized equations for the analysis of inhibitions of Michaelis-Menten and higher-order kinetic systems with two or more mutually exclusive and nonexclusive inhibitors. Eur J Biochem 115: 207-216.

Dang CV (2012) Links between metabolism and cancer. Genes Dev 26: 877-890.

Daye D, Wellen KE (2012) Metabolic reprogramming in cancer: unraveling the role of glutamine in tumorigenesis. Semin Cell Dev Biol 23: 362-369.

DeBerardinis RJ, Mancuso A, Daikhin E, Nissim I, Yudkoff M, Wehrli S, Thompson CB (2007) Beyond aerobic glycolysis: transformed cells can engage in glutamine metabolism that exceeds the requirement for protein and nucleotide synthesis. Proc Natl Acad Sci USA 104: 19345-19350.

DeBerardinis RJ, Thompson CB (2012) Cellular metabolism and disease: what do metabolic outliers teach us? Cell 148: 1132-1144.

Deer EL, Gonzalez-Hernandez J, Coursen JD, Shea JE, Ngatia J, Scaife CL, Firpo MA, Mulvihill SJ (2010) Phenotype and genotype of pancreatic cancer cell lines. Pancreas 39: 425-425.

Dowling RJ, Goodwin PJ, Stambolic V (2011) Understanding the benefit of metformin use in cancer treatment. BMC Med 9: 33.

Dranka BP, Zielonka J, Kanthasamy AG, Kalyanaraman B (2012) Alterations in bioenergetic function induced by Parkinson's disease mimetic compounds: lack of correlation with superoxide generation. J Neurochem 122: $941-951$

Dwarakanath B, Jain V (2009) Targeting glucose metabolism with 2-deoxyD-glucose for improving cancer therapy. Future Oncol 5: 581-585.

Fendt SM, Bell EL, Keibler MA, Davidson SM, Wirth GJ, Fiske B, Mayers JR, Schwab M, Bellinger G, Csibi A, Patnaik A, Blouin MJ, Cantley LC, Guarente L, Blenis J, Pollak MN, Olumi AF, Vander Heiden MG, Stephanopoulos G (2013) Metformin decreases glucose oxidation and increases the dependency of prostate cancer cells on reductive glutamine metabolism. Cancer Res 73: 4429-4438.

Ferreira LM, Hebrant A, Dumont JE (2012) Metabolic reprogramming of the tumor. Oncogene 31: 3999-4011.

Gatenby RA, Gillies RJ (2004) Why do cancers have high aerobic glycolysis? Nat Rev Cancer 4: 891-899.

Hartman DJ, Krasinskas AM (2012) Assessing treatment effect in pancreatic cancer. Arch Pathol Lab Med 136: 100-109.

Heinemann V, Haas M, Boeck S (2012) Systemic treatment of advanced pancreatic cancer. Cancer Treat Rev 38: 843-853.

Hidalgo M (2010) Pancreatic cancer. N Engl J Med 362: 1605-1617.

Iliopoulos D, Hirsch HA, Struhl K (2011) Metformin decreases the dose of chemotherapy for prolonging tumor remission in mouse xenografts involving multiple cancer cell types. Cancer Res 71: 3196-3201.

Jones NP, Schulze A (2012) Targeting cancer metabolism-aiming at a tumour's sweet-spot. Drug Discov Today 17: 232-241.

Kalariya NM, Shoeb M, Ansari NH, Srivastava SK, Ramana KV (2012) Antidiabetic drug metformin suppresses endotoxin-induced uveitis in rats. Invest Ophthalmol Vis Sci 53: 3431-3440.

Kim SA, Choi HC (2012) Metformin inhibits inflammatory response via AMPK-PTEN pathway in vascular smooth muscle cells. Biochem Biophys Res Commun 425: 866-872.

Kung HN, Marks JR, Chi JT (2011) Glutamine synthetase is a genetic determinant of cell type-specific glutamine independence in breast epithelia. PLoS Genet 7: e1002229.

Kurtoglu M, Lampidis TJ (2009) From delocalized lipophilic cations to hypoxia: blocking tumor cell mitochondrial function leads to therapeutic gain with glycolytic inhibitors. Mol Nutr Food Res 53: 68-75.

Le A, Rajeshkumar NV, Maitra A, Dang CV (2012) Conceptual framework for cutting the pancreatic cancer fuel supply. Clin Cancer Res 18: 4285-4290.

Liu H, Hu YP, Savaraj N, Priebe W, Lampidis TJ (2001) Hypersensitization of tumor cells to glycolytic inhibitors. Biochemistry 40: 5542-5547.

Maher JC, Savaraj N, Priebe W, Liu H, Lampidis TJ (2005) Differential sensitivity to 2-deoxy-D-glucose between two pancreatic cell lines correlates with GLUT-1 expression. Pancreas 30: e34-e39.

Maschek G, Savaraj N, Priebe W, Braunschweiger P, Hamilton K, Tidmarsh GF, De Young LR, Lampidis TJ (2004) 2-deoxy-D-glucose increases the efficacy of adriamycin and paclitaxel in human osteosarcoma and nonsmall cell lung cancers in vivo. Cancer Res 64: 31-34.

Menendez JA, Oliveras-Ferraros C, Cufi S, Corominas-Faja B, Joven J, Martin-Castillo B, Vazquez-Martin A (2012) Metformin is synthetically lethal with glucose withdrawal in cancer cells. Cell Cycle 11: 2782-2792.
Metallo CM, Gameiro PA, Bell EL, Mattaini KR, Yang J, Hiller K, Jewell CM, Johnson ZR, Irvine DJ, Guarente L, Kelleher JK, Vander Heiden MG, Iliopoulos O, Stephanopoulos G (2012) Reductive glutamine metabolism by IDH1 mediates lipogenesis under hypoxia. Nature $\mathbf{4 8 1}$ : 380-384.

Mullen AR, Wheaton WW, Jin ES, Chen PH, Sullivan LB, Cheng T, Yang Y, Linehan WM, Chandel NS, DeBerardinis RJ (2012) Reductive carboxylation supports growth in tumour cells with defective mitochondria. Nature 481: 385-388.

Nicholls DG, Darley-Usmar VM, Wu M, Jensen PB, Rogers GW, Ferrick DA (2010) Bioenergetic profile experiment using C2C12 myoblast cells. J Vis Exp 46: pii: 2511.

Pathania D, Millard M, Neamati N (2009) Opportunities in discovery and delivery of anticancer drugs targeting mitochondria and cancer cell metabolism. Adv Drug Deliv Rev 61: 1250-1275.

Pelicano H, Martin DS, Xu RH, Huang P (2006) Glycolysis inhibition for anticancer treatment. Oncogene 25: 4633-4646.

Regel I, Kong B, Raulefs S, Erkan M, Michalski CW, Hartel M, Kleeff J (2012) Energy metabolism and proliferation in pancreatic carcinogenesis. Langenbecks Arch Surg 397: 507-512.

Salani B, Marini C, Rio AD, Ravera S, Massollo M, Orengo AM, Amaro A, Passalacqua M, Maffioli S, Pfeffer U, Cordera R, Maggi D, Sambuceti G (2013) Metformin impairs glucose consumption and survival in Calu-1 cells by direct inhibition of hexokinase-II. Sci Rep 3: 2070.

Scott DA, Richardson AD, Filipp FV, Knutzen CA, Chiang GG, Ronai ZA, Osterman AL, Smith JW (2011) Comparative metabolic flux profiling of melanoma cell lines: beyond the Warburg effect. J Biol Chem 286: 42626-42634.

Segal ED, Yasmeen A, Beauchamp MC, Rosenblatt J, Pollak M, Gotlieb WH (2011) Relevance of the OCT1 transporter to the antineoplastic effect of biguanides. Biochem Biophys Res Commun 414: 694-699.

Son J, Lyssiotis CA, Ying H, Wang X, Hua S, Ligorio M, Perera RM, Ferrone CR, Mullarky E, Shyh-Chang N, Kang Y, Fleming JB, Bardeesy N, Asara JM, Haigis MC, DePinho RA, Cantley LC, Kimmelman AC (2013) Glutamine supports pancreatic cancer growth through a KRAS-regulated metabolic pathway. Nature 496: 101-105.

Strimpakos A, Saif MW, Syrigos KN (2008) Pancreatic cancer: from molecular pathogenesis to targeted therapy. Cancer Metastasis Rev 27: 495-522.

Tennant DA, Duran RV, Gottlieb E (2010) Targeting metabolic transformation for cancer therapy. Nat Rev Cancer 10: 267-277.

Tucker ON, Dennenberg AJ, Yang EK, Zhang F, Teng L, Daly JM, Soslow RA, Masferrer JL, Woener BM, Koki AT, Fahey 3rd TJ (1999) Cyclooxygenase2 expression is up-regulated in human pancreatic cancer. Cancer Res 59: 987-990.

Vander Heiden MG, Cantley LC, Thompson CB (2009) Understanding the Warburg effect: the metabolic requirements of cell proliferation. Science 324: 1029-1033.

Viollet B, Guigas B, Sanz GN, Leclerc J, Foretz M, Andreelli F (2012) Cellular and molecular mechanisms of metformin: an overview. Clin Sci (Lond) 122: $253-270$.

Wang DS, Jonker JW, Kato Y, Kusuhara H, Schinkel AH, Sugiyama Y (2002) Involvement of organic cation transporter 1 in hepatic and intestinal distribution of metformin. J Pharmacol Exp Ther 302: 510-515.

Ward PS, Thompson CB (2012) Metabolic reprogramming: a cancer hallmark even Warburg did not anticipate. Cancer Cell 21: 297-308.

Wenner CE (2012) Targeting mitochondria as a therapeutic target in cancer. J Cell Physiol 227: 450-456.

Wise DR, Thompson CB (2010) Glutamine addiction: a new therapeutic target in cancer. Trends Biochem Sci 35: 427-433.

Zannella VE, Pra AD, Muaddi H, McKee TD, Stapleton S, Sykes J, Glicksman R, Chaib S, Zamiara P, Milosevic M, Wouters BG, Bristow RG, Koritzinsky M (2013) Reprogramming metabolism with metformin improves tumor oxygenation and radiotherapy response. Clin Cancer Res 19: 6741-6750.

This work is published under the standard license to publish agreement. After 12 months the work will become freely available and the license terms will switch to a Creative Commons AttributionNonCommercial-Share Alike 3.0 Unported License. 